\title{
Obesity moderates the complex relationships between inflammation, oxidative stress, sleep quality and depressive symptoms
}

\author{
Alanna V. Rigobon ${ }^{1}$, Thirumagal Kanagasabai ${ }^{2}$ and Valerie H. Taylor $34^{3 *}$
}

\begin{abstract}
Background: The relationship between obesity and depression is complex. This study assessed the impact of body mass index (BMI) on the link between BMI, inflammation, oxidative stress, sleep quality and self-reported depressive symptoms.

Methods: We used data from the U.S. National Health and Nutritional Examination Survey 2005-2008 cycles ( $n=9133$; $\geq 20 y$ ). Depressive symptoms and sleep quality were determined from questionnaires. C-reactive Protein (CRP) was used as a biomarker of inflammation and $\gamma$-glutamyltransferase was used to assess oxidative stress. The relationship between depressive symptoms, sleep quality, and biomarkers were assessed with regression models. The moderating effects of BMI and sex were tested.

Results: BMI was a significant moderator of the relationship between $\gamma$-glutamyltransferase and depressive symptoms $(p=0.02)$, but not CRP or sleep quality. Higher BMl increased odds of depressive symptoms in women (OR ( $95 \% \mathrm{Cl}): 3.92$ (1.85-8.30) for BMI $\geq 25$ to $<30 \mathrm{~kg} / \mathrm{m}^{2} ; 3.17(1.53-6.58)$ for BMI $\geq 30$ to $<35 \mathrm{~kg} / \mathrm{m}^{2}$; and $7.38(2.11-25.76)$ for BMI $\geq 35 \mathrm{~kg} / \mathrm{m}^{2}$ ). BMI was also a significant moderator of $y$-glutamyltransferase levels in those with vs without depressive symptoms. Those with depressive symptoms had $24 \%$ poorer sleep quality compared to those without depressive symptoms after adjusting for inflammation, oxidative stress and other confounders.

Conclusions: The link between oxidative stress and depressive symptoms may be particularly relevant for females and people living with obesity. People with depressive symptoms also have a substantial reduction in sleep quality. Thus, research should examine these relationships prospectively to inform and improve the mental health of the adult population in developed countries.
\end{abstract}

Keywords: Body mass index (BMI), Obesity, Sleep, Inflammation, Oxidative stress, Depression

\section{Background}

Evidence has established a bidirectional link between depression and obesity $[1,2]$. Obesity is associated with an increased vulnerability for depression, secondary to factors such as chronic pain, reduced mobility and issues related to societal bias and stigma [1, 3, 4]. For example, obesity places increased workload on joints and increases pro-inflammatory cytokines that promote further

\footnotetext{
* Correspondence: valerie.taylor@wchospital.ca

${ }^{3}$ Women's College Research Institute, University of Toronto, Toronto, Canada ${ }^{4}$ Women's College Hospital, 76 Grenville Street Room E947, Toronto, ON M5S 1B2, Canada

Full list of author information is available at the end of the article
}

joint destruction and arthritis, one of the most common causes of chronic pain and reduced mobility in adults [5-7]. Conversely, depression increases the likelihood of obesity, given that decreased activity, fatigue and appetite changes are core symptoms $[1,8]$. Studies examining the link between these conditions also suggest a common inflammatory pathway $[9,10]$. Patients with depression show elevated levels of pro-inflammatory cytokines, acute phase reactants such as C-reactive protein (CRP), chemokines, and cell adhesion molecules [11]. Concomitantly, treatment studies suggest that inhibiting the inflammatory cytokine tumour necrosis factor (TNF) may improve depressive symptoms in patients with high

(C) The Author(s). 2018 Open Access This article is distributed under the terms of the Creative Commons Attribution 4.0 International License (http://creativecommons.org/licenses/by/4.0/), which permits unrestricted use, distribution, and 
baseline inflammatory biomarkers [12, 13]. Obesity also has an inflammatory component, and much of its clinical pathology has been linked to obesity-associated chronic inflammation of white adipose tissue and the resultant increased circulating concentrations of inflammatory markers $[12,14]$. Although a common inflammatory pathway has been hypothesized to explain some of the depression-obesity confluence, evidence of a biological link between obesity and depression is conflicting.

A second biological association between depression and obesity relates to oxidative stress, a condition defined as a persistent imbalance between antioxidant and pro-oxidant processes, where the end result is excessive production of reactive oxygen species (ROS) and reactive nitrogen species (RNS) [15]. Increased levels of oxidative stress biomarkers are observed in both obese and depressed states [16, 17]. Recent studies show that oxidative stress in combination with the pro-inflammatory mechanism plays an important role in the development of a number of psychiatric disorders [18, 19]. This has led to the hypothesis that the combination of these pathways forms the pathophysiological basis of depression.

Sleep quality is also thought to play a role in the proinflammatory state seen in depression and obesity. Both experimental and clinical studies show a bidirectional association between sleep and inflammation [20]. Sleep loss, for instance, is known to induce proinflammatory markers CRP, Interleukin-6 (IL-6) and TNF that are associated with depression and obesity [21-23]. Proinflammatory cytokines also influence sleep, and the administration of infectious agents can fragment sleep via cytokine expression $[24,25]$. Restoration of sleep decreases the induction of inflammatory pathways, reduces symptoms of depression, and attenuates gains in fat mass [26, 27]. These findings suggest that sleep-related mechanisms are implicated in the immunologic pathways linking depression and obesity.

Indeed, multifaceted factors link the etiology of both obesity and depression. Our study aims to: (1) assess the moderating effect of obesity on the associations of depressive symptoms with inflammation (i.e. CRP), oxidative stress (i.e. $\gamma$-glutamyltransferase), and sleep quality; and, (2) determine which of these factors increase the odds of having depressive symptoms in a nationally representative sample of U.S. adults.

\section{Methods}

\section{Participants}

The U.S. National Health and Nutrition Examination Survey (NHANES) is a series of nationally representative cross-sectional studies designed to assess the health and nutritional status of the non-institutionalized civil population [28]. NHANES uses multi-stage, unequal probability, and cluster sampling methods to recruit its participants. Data is collected from personal interviews, standardized physical examinations, and laboratory samples by trained professionals. Ethical approval was provided by the National Center for Health Studies Ethics Review Board for the NHANES, and informed consent was obtained from all participants. Approximately 10,000 people are sampled bi-annually, and the initial sample of our analysis included 20,497 individuals from the NHANES 2005-2008 cycles. We excluded from our analysis individuals with missing sleep quality variables $(n=18)$ or depression data $(n=1371)$, pregnancy $(n=392)$, and individuals less than 20 years old, $(<20$ y; $n=9583)$. We excluded children and adolescents from our analysis since their BMI categorization is based on percentile, and their information on certain behavioural measures (e.g., smoking and alcohol intake) are restricted. Our final analytic sample was 9133 participants.

\section{BMI}

We used body mass index (BMI; $\mathrm{kg} / \mathrm{m}^{2}$ ) to measure the degree of obesity. BMI was categorized as $<18.5, \geq 18.5$ to $<25, \geq 25$ to $<30, \geq 30$ to $<35$, and $\geq 35 \mathrm{~kg} / \mathrm{m}^{2}$ from the continuous BMI variable provided by NHANES. Standing height was measured as the maximum vertical size using a Stadiometer to the nearest $\mathrm{mm}$. Weight was determined with a Toledo digital scale in pounds, which was then converted to kilograms with the automated system by NHANES.

\section{Sleep quality}

Sleep habits of participants were obtained from 6 questions ["In the past month, how often did you have trouble falling asleep?", "In the past month, how often did you wake up during the night and had trouble getting back to sleep?", "In the past month, how often did you wake up too early in the morning and were unable to get back to sleep?", "In the past month, how often did you feel unrested during the day, no matter how many hours of sleep you have had?", "In the past month, how often did you feel excessively or overly sleepy during the day?", and "In the past month, how often did you not get enough sleep?"] from the Sleep Disorders Questionnaire. Only 2005-2008 NHANES cycles' questionnaires contain these questions, and thus, our analysis is restricted to these cycles. The Sleep Disorders Questionnaire was administered to participants aged $\geq 16$, who reported their typical sleep habits on weekdays or workdays over the past month, and responses were coded as $0=$ "Never", 1 $=$ "Rarely (1 time a month)", $2=$ "Sometimes (2-4 times a month)", 3 = "Often (5-15 times a month)", 4="Almost always (16-30 times a month)". Individual responses from the 6 questions were then summed to obtain a global sleep quality score for each participant [29] with a higher sleep quality score indicating lower sleep quality. 


\section{Depressive symptoms and severity}

The Patient Health Questionnaire (PHQ-9) was used to estimate prevalence and severity of depressive symptoms [30]. The presence of depressive symptoms was conferred based on participants' responses to 9 depression-related symptoms over the last 2 weeks. Responses to each question were: "Not at all", "Several days", "More than half the days", and "Nearly every day" (scored as $0,1,2,3$, respectively), which were summed and categorized: depressive symptom severity ("None": 0-4; "Minimal": 5-9; "Mild": 10-14; "Moderate": 15-19; and "Severe": 20-27); Participants with none to mild symptom severity (i.e., score of $0-14)$ were categorized as having "no symptoms of depression", and participants with moderate to severe depression severity (i.e., score of 15-27) were categorized as having "depressive symptoms" [31, 32].

\section{Inflammation and oxidative stress}

Laboratory measures of CRP (nM) and $\gamma$-glutamyltran sferase $(\mathrm{U} / \mathrm{L})$ were used to assess inflammation and oxidative stress, respectively [33, 34]. The average time between initial participant interview and medical examination center visit for laboratory collection was 2 weeks [35]. Description of the laboratory methodology, data processing, and quality assurance details for the biomarkers are available on NHANES's website [36]. Briefly, CRP was measured in frozen serum using Nephelometry at the University of Washington Medical Center. Blood was collected using regular or serum-separator Vacutainers, and the serum was separated from the cells within $60 \mathrm{~min}$ of the collection. The samples were stored in tightly sealed vials to prevent desiccation and frozen at $\leq 20{ }^{\circ} \mathrm{C}$ until particle-enhanced assay could be performed. The assay uses latex particles coated with mouse monoclonal anti-CRP antibodies to quantify the presence of CRP in the samples as they form antigen-antibody complexes with the latex particles. $\gamma$-glutamyltransferase was measured in Refrigerated Serum using Beckman Synchron LX20 by Collaborative Laboratory Services, L.L.C. Separated samples were frozen at -15 to $-20{ }^{\circ} \mathrm{C}$ and thawed until LX20 analysis. The LX20 uses an enzymatic method to quantify $\gamma$-glutamyltransferase activity at an absorbance of $410 \mathrm{~nm}$. Participant fasting was not required for CRP or $\gamma$-glutamyltransferase sample collection.

\section{Covariates}

Age, sex, ethnicity, education, income, smoking, alcohol intake, and recreational physical activity level were considered as potential confounders [37]. Age was also categorized as 20 to $<40,40$ to $<65$, and $\geq 65$ y. Ethnicity was self-reported and categorized as non-Hispanic White, non-Hispanic Black, Mexican American, and Other. Income was also self-reported and categorized as $<\$ 20,000$, $\$ 20,000$ to 44,999 , and $\geq \$ 45,000 ;$ and, education categorized as <high school, high school, and at least some college or above. Alcohol intake was categorized as $<3$ or $\geq 3$ drinks per day. Smoking was categorized as current (if smoking now), past (if they smoked $\geq 100$ cigarettes in a lifetime) or never (if smoked $<100$ cigarettes in a lifetime) [38]. Further, recreational physical activity level was calculated using the metabolic equivalent (MET) score assigned for each activity by NHANES and categorized as "inactive" (no reported recreational physical activity data), "somewhat active" (<500 MET-min/week) and "active" ( $\geq 500$ MET-min/week).

\section{Statistical analyses}

Mean and 95\% confidence interval (CI) for continuous variables, and percentage and 95\% CI for categorical variables were determined according to presence or absence of depressive symptoms. Differences in demographic and behavioural characteristics of participants were assessed by independent $\mathrm{t}$-test and $x^{2}$ analyses, as appropriate. PROC SURVEYLOGISTIC was used to assess the association of depressive symptoms $(0,1)$ with CRP, $\gamma$-glutamyltransferase, and sleep quality. PROC SURVEYREG was used to estimate CRP, $\gamma$-glutamyltransferase, and sleep quality in adults with vs without symptoms of depression. The moderating effect of BMI and sex on these associations were tested with interaction terms. Confounding variables adjusted in our models include age, sex, ethnicity, education, income, smoking, alcohol intake, and recreational physical activity. When appropriate, CRP, $\gamma$-glutamyltransferase, and sleep quality were also considered as confounders. All analyses are weighted to represent the U.S. adult population using SAS v9.4 (Cary, NC, U.S.A). Statistical significance was set at $\alpha<0.05$.

\section{Results}

Table 1 describes the US adult population by the presence or absence of self-reported depressive symptoms. Those with depressive symptoms were more likely to be middle-aged ( $\geq 40$ to $<65 \mathrm{y}$ ), women, Non-Hispanic Blacks, have lower education and income, and be current smokers. In general, higher severity of depressive symptoms was associated with higher inflammation and oxidative stress, and lower sleep quality (Fig. 1). Obesity was a significant moderator of the relationship between depressive symptoms and $\gamma$-glutamyltransferase $(p=0.02)$, but not CRP $(p=0.43)$ or sleep quality $(p=0.93)$. Rather, the later relationships were strongly confounded by sex (i.e., higher in women compared to men), income, smoking status, and physical activity (Table 2). The moderating effect of obesity on the former relationship between $\gamma$-glutamyltransferase and depressive symptoms was very weak (Table 3), and it may only be important in women. Indeed, of the covariates considered in our analysis, only being a woman was significantly associated with higher 
Table 1 Characteristics of the US adult population ( $\geq 20 \mathrm{y})$

\begin{tabular}{|c|c|c|c|}
\hline Characteristics & No Depressive Symptoms $(n=8888)$ & Depressive Symptoms $(n=245)$ & $P$ value \\
\hline Age (Mean (95\% Cl)) & $46.9(46.1,47.8)$ & $46.6(45.1,48.1)$ & NS \\
\hline \multicolumn{4}{|l|}{ Age categories (\% (95\% CI)) } \\
\hline$\geq 20$ to $<40 y$ & $36.7(34.8,38.7)$ & $31.1(23,39.2)$ & \multirow[t]{3}{*}{$<0.05$} \\
\hline$\geq 40$ to $<65 y$ & $46.0(44.3,47.7)$ & $61.8(52.1,71.5)$ & \\
\hline$\geq 65$ y & $17.3(15.5,19.0)$ & $7.1(4.0,10.2)$ & \\
\hline \multicolumn{4}{|l|}{ Sex } \\
\hline Men & $49.6(48.6,50.6)$ & $31.7(25.2,38.1)$ & \multirow[t]{2}{*}{$<0.05$} \\
\hline Women & $50.4(49.4,51.4)$ & $68.3(61.9,74.8)$ & \\
\hline \multicolumn{4}{|l|}{ Ethnicity } \\
\hline Non-Hispanic White & $72.1(67.6,76.6)$ & $65.0(55.9,74.2)$ & \multirow[t]{4}{*}{$<0.05$} \\
\hline Non-Hispanic Black & $10.8(8.1,13.5)$ & $17.3(10.5,24.2)$ & \\
\hline Mexican American & $7.9(6.1,9.8)$ & $7.3(3.7,10.9)$ & \\
\hline Others & $9.2(7.3,11)$ & $10.3(5.1,15.5)$ & \\
\hline \multicolumn{4}{|l|}{ Education } \\
\hline$<$ High school & $18.2(16,20.4)$ & $29.5(23.4,35.5)$ & \multirow[t]{3}{*}{$<0.05$} \\
\hline High school & $25.0(23.3,26.7)$ & $31.0(21.6,40.4)$ & \\
\hline College & $56.8(53.4,60.1)$ & $39.5(30.6,48.4)$ & \\
\hline \multicolumn{4}{|l|}{ Income } \\
\hline$<\$ 20,000$ & $15.5(13.8,17.3)$ & $35.5(30.5,40.6)$ & \multirow[t]{3}{*}{$<0.05$} \\
\hline$\$ 20,000-44,999$ & $29.8(27.6,32.0)$ & $38.8(30.7,47.0)$ & \\
\hline$\geq \$ 45,000$ & $54.7(51.4,57.9)$ & $25.6(18.4,32.9)$ & \\
\hline \multicolumn{4}{|l|}{ Smoking } \\
\hline None & $51.6(49.4,53.7)$ & $38.4(28.9,47.9)$ & \multirow[t]{3}{*}{$<0.05$} \\
\hline Current & $23.2(21.4,25)$ & $45.1(35.9,54.2)$ & \\
\hline Past & $25.2(23.8,26.6)$ & $16.5(11.2,21.9)$ & \\
\hline \multicolumn{4}{|l|}{ Alcohol Intake } \\
\hline$<3$ drinks per day & $62.3(60,64.6)$ & $56.1(44.9,67.3)$ & \multirow[t]{2}{*}{ NS } \\
\hline$\geq 3$ drinks per day & $37.7(35.4,40)$ & $43.9(32.7,55.1)$ & \\
\hline \multicolumn{4}{|c|}{ Recreational Physical Activity } \\
\hline None reported & $66.2(62.6,69.9)$ & $84.4(77.8,91)$ & \multirow[t]{3}{*}{$<0.05$} \\
\hline$<500$ MET-min/week & $11.4(10.1,12.7)$ & $6.1(2,10.2)$ & \\
\hline$\geq 500$ MET-min/week & $22.3(19.7,24.9)$ & $9.5(5.4,13.6)$ & \\
\hline \multicolumn{4}{|l|}{ BMI categories $\left(\mathrm{kg} / \mathrm{m}^{2}\right)$} \\
\hline$<18.5$ & $1.6(1.2,2.0)$ & $1.1(0.0,2.2)$ & \multirow[t]{5}{*}{ NS } \\
\hline$\geq 18.5-<25$ & $30.7(29.0,32.4)$ & $24.3(17.5,31.2)$ & \\
\hline$\geq 25-<30$ & $33.7(32.5,35.0)$ & $31.5(25.0,38.0)$ & \\
\hline$\geq 30-<35$ & $19.4(18.5,20.4)$ & $21.9(14.5,29.3)$ & \\
\hline$\geq 35$ & $14.5(13.2,15.9)$ & $21.1(14.2,28.1)$ & \\
\hline
\end{tabular}

Mean $(95 \% \mathrm{Cl})$ for continuous variables and \% $(95 \% \mathrm{Cl})$ for categorical variables. Responses from the PHQ-9 were summed and categorized as None $(0-4)$, Minimal (5-9), Mild (10-14), Moderate (15-19), and Severe (20-27). Moderate-to-Severe was categorized as Depressive Symptoms. Sleep Quality are sum of responses for 6 sleep habit questions from the Sleep Disorder Questionnaire. $p<0.05$, two-sided; t-test or Chi-square, as appropriate. NS not significant. Sum of weights $=190,972,201$

odds of having depressive symptoms and the magnitude of this association increased with BMI. Sex was not a moderator of the relationship between depressive symptoms and $\gamma$-glutamyltransferase $(p=0.31)$.
Additionally, compared to participants without depressive symptoms, participants with symptoms had 24.65 nM (95\% CI: 5.25, 44.06) higher CRP levels. This association also remained significant after adjustments 

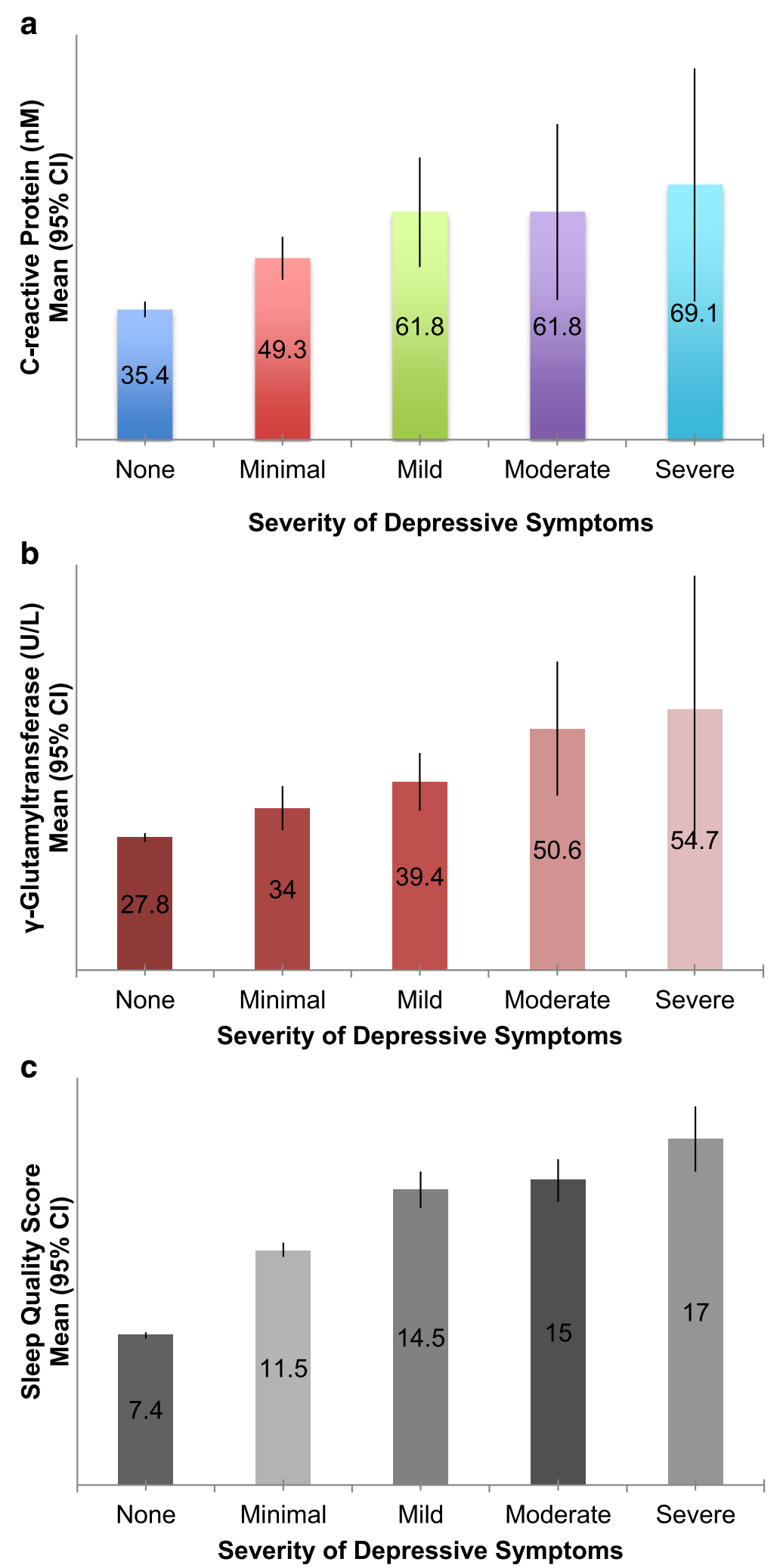

Fig. 1 Higher severity of depressive symptom is associated with higher inflammation (a), oxidative stress (b), and lower sleep quality (c) 
Table 2 Multivariable model for C-reactive Protein and sleep quality and their association with depressive symptoms

\begin{tabular}{llr}
\hline Variable & OR $(95 \% \mathrm{Cl})$ & \multicolumn{1}{c}{ Sleep Quality } \\
\cline { 2 - 3 } & C-reactive Protein & - \\
\hline C-reactive Protein & $1.001(1.001,1.002)$ & $1.190(1.151,1.231)$ \\
Sleep Quality & - & $1.108(0.918,1.339)$ \\
BMI & $1.130(0.958,1.333)$ & $1.004(0.989,1.019)$ \\
Age & $0.999(0.986,1.012)$ & $1.657(1.098,2.499)$ \\
Sex & $2.336(1.562,3.492)$ & $0.967(0.808,1.158)$ \\
Ethnicity & $0.910(0.726,1.141)$ & $0.947(0.732,1.225)$ \\
Education & $0.974(0.763,1.242)$ & $0.472(0.380,0.587)$ \\
Income & $0.418(0.332,0.526)$ & $0.677(0.515,0.889)$ \\
Smoking & $0.789(0.630,0.989)$ & $1.321(0.739,2.362)$ \\
Alcohol & $1.415(0.794,2.520)$ & $0.658(0.480,0.903)$ \\
Recreational Physical Activity & $0.624(0.470,0.828)$ &
\end{tabular}

OR is the odds of having depressive symptoms adjusted for BMI, age, sex, ethnicity, education, income, smoking, alcohol, and recreational physical activity. All variables except for age were modeled categorically

for age, sex, ethnicity, education, income, smoking, alcohol, and recreational physical activity to $12.13 \mathrm{nM}$ $(1.43,22.82)$ but became non-significant following adjustments for BMI $(9.11 \mathrm{nM}(-0.31,18.53))$ and sleep quality $(6.02 \mathrm{nM}(-3.45,15.49))$. BMI did not moderate of the association between CRP levels or sleep quality and depressive symptoms. The association between $\gamma$-glutamyltransferase and depressive symptoms was, however, moderated by BMI $(p=0.04)$. Compared to participants without depressive symptoms and BMI $\geq 18.5$ to $<25$, participants with depressive symptoms had significantly higher $\gamma$-glutamyltransferase levels: $2.41 \mathrm{U} / \mathrm{L}(0.88,3.95)$ for $\mathrm{BMI} \geq 25$ to $<30$; 8.67 (2.46, 14.87) for $\mathrm{BMI} \geq 30$ to $<25$, and 12.35 (8.33, 16.37) for $\mathrm{BMI} \geq 35$, respectively) after adjusting for age, sex, ethnicity, education, income, smoking, alcohol, recreational physical activity and sleep quality. Finally, compared to participants without depressive symptoms, participants with depressive symptoms had significantly poorer sleep quality (i.e., the latter group had 5.84 (4.61, 7.06) higher sleep quality score out of a possible 24 points or $24.3 \%$ poorer sleep quality) after adjusting for sex, ethnicity, education, income, smoking, alcohol, recreational physical activity, CRP and $\gamma$-glutamyltransferase.

\section{Discussion}

Results from this study are consistent with previous evidence that suggests inflammatory and oxidative stress are associated with depressive symptoms, obesity, and sleep disturbances [9, 20, 23, 39]. Indeed, we found that participants with self-reported depressive symptoms had higher inflammation and oxidative stress biomarkers, and poorer sleep quality compared to those without depressive symptoms. Our study also suggests that these

Table 3 Multivariable model for the relationship between $\gamma$-glutamyltransferase and the odds of having depressive symptoms, stratified by BMI categories

\begin{tabular}{|c|c|c|c|c|}
\hline \multirow[t]{3}{*}{ Variable } & \multicolumn{4}{|l|}{ OR $(95 \% \mathrm{Cl})$} \\
\hline & \multicolumn{4}{|c|}{ BMI categories $\left(\mathrm{kg} / \mathrm{m}^{2}\right)$} \\
\hline & $\geq 18.5-<25$ & $\geq 25-<30$ & $\geq 30-<35$ & $\geq 35$ \\
\hline Y-glutamyltransferase & $1.009(1.002,1.017)$ & $1.004(1.001,1.007)$ & $1.004(0.999,1.009)$ & $1.003(1.000,1.005)$ \\
\hline Age & $0.996(0.968,1.025)$ & $0.993(0.972,1.016)$ & $0.971(0.951,0.991)$ & $1.022(0.989,1.057)$ \\
\hline Sex & $1.218(0.519,2.858)$ & $3.916(1.848,8.297)$ & $3.168(1.525,6.580)$ & $7.377(2.113,25.759)$ \\
\hline Ethnicity & $1.192(0.783,1.813)$ & $0.586(0.409,0.839)$ & $0.663(0.419,1.051)$ & $1.213(0.692,2.125)$ \\
\hline Education & $0.963(0.493,1.879)$ & $0.895(0.520,1.540)$ & $0.795(0.441,1.433)$ & $1.493(0.913,2.442)$ \\
\hline Income & $0.668(0.390,1.146)$ & $0.387(0.252,0.593)$ & $0.406(0.222,0.744)$ & $0.256(0.169,0.389)$ \\
\hline Smoking & $0.854(0.441,1.654)$ & $0.637(0.410,0.990)$ & $0.882(0.564,1.379)$ & $0.780(0.438,1.390)$ \\
\hline Alcohol & $2.242(0.765,6.569)$ & $1.186(0.394,3.568)$ & $0.955(0.442,2.066)$ & $0.872(0.335,2.268)$ \\
\hline Recreational Physical Activity & $0.709(0.421,1.196)$ & $0.709(0.378,1.332)$ & $0.171(0.018,1.631)$ & $0.607(0.267,1.377)$ \\
\hline
\end{tabular}

$\mathrm{OR}$ is the odds of having depressive symptoms adjusted for age, sex, ethnicity, education, income, smoking, alcohol, and recreational physical activity. All variables except for age were modeled categorically 
relationships are lower than in some clinical studiesthe lower effect sizes could be partly explained by the wider variations in both the exposure and outcome that are commonly found at the population level or the self-reported nature of depressive symptoms as an outcome. It has previously been hypothesized that the link between systemic inflammation, oxidative stress, and depression are associated with BMI $[9,23,40]$. We found a moderating effect for BMI on the relationship between y-glutamyltransferase and depressive symptoms, which augments current literature. The novelty of this study lies in our sex-specific findings, which suggest the link between oxidative stress and depressive symptoms may be particularly relevant for females. Female sex was a significant driver of this relationship, and higher BMI exacerbated the association between y-glutamyltransferase and depressive symptoms in women.

\section{Inflammation}

The relationship between increased inflammatory biomarkers and depression has been well documented in the literature $[23,41]$. In accordance with previous findings, our results showed slightly higher odds of depressive symptoms for inflammatory marker CRP. The lower magnitude of the association in our study could be due to multiple factors, including the self-reported nature of our outcome and the population level study design that is susceptible to higher variability. However, previous studies have implicated obesity as a possible moderating factor in this relationship $[9,23,40]$. Increased body mass appears to account for a portion of the relationship between depression and increased inflammatory markers $[9,23]$. Consistent with this hypothesis, Miller and colleagues (2002) noted a synergistic relationship between depression and obesity with respect to CRP levels [40]. Indeed, depressive symptoms can facilitate weight gain over time as a result of sedentary behaviour $[40,42]$. As fat cells accumulate, they produce leptin which acts to upregulate the expression of IL-6, thereby stimulating CRP, and promoting systemic inflammation [43]. Depression and fat mass are also thought to induce inflammation through their association with heightened Hypothalamic Pituitary Axis and Sympathetic Nervous System activity [44]. In particular, cytokines released in these inflammatory states can disrupt glucocorticoid receptor function, causing increased glucocorticoid resistance, and impaired negative regulation of Corticotropin-Releasing Hormone (CRH) by glucocorticoids [45]. Hyperactivity of $\mathrm{CRH}$ thereby leads to increased HPA and sympathetic response, further promoting inflammatory activation [11].

BMI did not moderate the relationship between inflammatory biomarkers and depressive symptoms in our study. However, we only tested one marker of inflammation (CRP) because other robust markers of inflammation (e.g.,
IL- 6 and TNF- $\alpha$ ) are not available in the NHANES data set, and the current utility of these inflammatory markers in depression is unclear [46]. CRP is a commonly used biomarker in the study of depression and obesity, and shows positive associations with both disease states [23, 47]. Further research is needed to examine the moderating and mediating effects of BMI on depression with additional biomarkers, e.g., malondialdehyde, F2-isoprostanes, 8-hydroxy 2 '-deoxyguanosine, neuropeptide $\mathrm{Y}$, adiponectin, IL-6, TNF- $\alpha$, and IL-17.

\section{Oxidative stress}

Previous studies exploring the relationship between oxidative stress and depression have used a variety of biomarkers to assess oxidative stress including F2 isoprostanes, 8-hydroxydeoxyguanosine and protein carbonyls [48]. The majority of these studies demonstrate an association between increased levels of oxidative stress markers and depression [17, 48]. The current study is the second to examine this relationship using y-glutamyltransferase and provides support for an association between depressive symptoms and increased oxidative stress for this biomarker [34], y-glutamyltransferase levels correlate with the body's total antioxidant capacity and this biomarker has been used as a surrogate marker of oxidative stress in other NHANES-based analyses [34].

The depressed state is associated with compromised antioxidant responses, activated oxidative/nitrosative stress pathways and damage to fatty acids, DNA, and mitochondria [49]. It has been hypothesized that activation of oxidative stress pathways may increase susceptibility to neurodegeneration, and depression, as cells in the brain are at an increased vulnerability due to a high metabolic rate, low antioxidant levels, and an abundance of polyunsaturated fatty acids prone to oxidation [50, 51]. Our study was limited to the assessment of associations between these disease states, and further studies would be helpful to better delineate causality in order to understand underlying pathogenesis.

Oxidative stress pathways may be particularly relevant in individuals with obesity. Obesity is associated with hyperglycemia, increased muscle activity, elevated tissue lipid levels, inadequate antioxidant defenses, chronic inflammation, endothelial ROS production, and hyperleptinemia, which are all thought to contribute to oxidative stress [39]. Our results suggest a significant moderating effect of BMI on the relationship between $y$-glutamyltransferase and depression. Adipose tissue releases a host of immune factors that increase oxidative stress, and the accumulation of abdominal fat is specifically associated with elevations in lipid and protein peroxidation $[52,53]$. It is possible that oxidative stress induced by fat tissue accumulation may interact 
or synergize with the increased oxidative response seen in depression, leading to differential effects in depressive patients according to BMI or adiposity classifications.

A novelty of our study lies with our sex-specific findings for the relationship between oxidative stress, obesity and depressive symptoms, which may be more relevant for females who show increased vulnerability to these disease states [54-56]. Clinical studies examining the effects of sex on oxidative stress in depressed patients are very limited and present controversial results [56-58]. A recent cross-sectional study in a small sample $(n=54)$ of healthy college students suggests the presence of a mild association between depressive symptoms and oxidative stress that is only present in females [58]. Females with depression also show increased levels of oxidative stress compared to non-depressed females [59]. Other researchers suggest that after sample stratification by sex, there is no association between oxidative stress parameters and clinical diagnosis of depression for females or males [60]. The reasons for inconsistency among these limited and early findings are not clear. It is possible that variation in the way depression is defined, i.e. self-reported accounts vs. clinical diagnoses, may affect the oxidative stress-sex relationship observed.

Results from our multivariable analysis corroborated findings by Matsushita and colleagues (2013) to suggest that female sex is a significant predictor of depressive symptoms for $\gamma$-glutamyltransferase [58]. Females showed increased odds of depressive symptoms and $\gamma$-glutamyltransferase across BMI categories and demonstrated a trend for increased odds of depressive symptoms and $\gamma$-glutamyltransferase with increasing BMI. Abdelkrim and colleagues (2015), suggest a variety of factors may contribute to these differences including fat tissue and distribution, hormonal differences, genetics, and variations in lifestyle [57]. Most relevant to our study is that an increased proportion of fat tissue in females may affect the oxidant/antioxidant balance. This is in line with research that suggests females have decreased antioxidant levels which are associated with adiposity/waist circumference [61].

\section{Sleep quality}

Sleep quality represents an additional factor implicated in the inflammatory pathways that underlie depression. Studies from both healthy and depressed populations show an association between higher CRP levels and characteristics of sleep including disturbance, duration and quality [62-64]. Independent of inflammation, oxidative stress, and common covariates that affect the relationship between sleep quality and depression, our study is the first to report that people with depression have substantially reduced sleep quality. However, the definition of sleep quality remains unclear, and it may vary among various subpopulations. We also did not assess sleep latency, as this information was truncated at $60 \mathrm{~min}$ in both NHANES 2005-2005 and 2006-2008 datasets. Additional studies are needed to identify the appropriate dose-response relationship between sleep quality and inflammatory biomarkers in depression. These should also involve other data sets, which include objective measures of sleep quality such as sleep latency and efficiency.

\section{Strengths and limitations}

Limitations of the study should be acknowledged. First, a cross-sectional design does not enable us to infer causality, and results must be interpreted cautiously. Additional longitudinal studies are needed to determine temporal sequences. Second, the relationship between obesity and depressive symptoms was examined using BMI, while indicators of central adiposity, including waist circumference, often demonstrate stronger associations with inflammation and oxidative stress [57, 61]. Third, we were unable to account for participants taking antidepressant medications, which may alter the levels of inflammatory and oxidative stress biomarkers [65, 66]. Fourth, depressive symptoms were based on self-reported questionnaires in the NHANES dataset and do not represent clinical diagnoses. All self-reported variables in our analysis are subject to recall and response biases. The majority of population-based studies examining the relationships between obesity and depression have also used questionnaire data to assess depression [1]. Fifth, there are an abundance of acute and chronic conditions, which may affect inflammatory markers studied, however it was not feasible to include all of these in the current study. Despite these limitations, our study provides novel evidence for a moderating effect of BMI on the relationship between oxidative stress and depressive symptoms in a large, nationally representative population. Further, NHANES provides sampling weights which take into account survey nonresponse, oversampling, post-stratification and sampling error to enable generalization of findings to the non-institutionalized civil adult population in the U.S.

\section{Conclusions}

This study suggests that BMI may moderate the relationship between oxidative stress and depressive symptoms, particularly amongst females. We also found that people with depressive symptoms have substantially lower sleep quality independent of systemic inflammation and oxidative stress. Future work should be directed at examining this relationship longitudinally and with additional inflammatory and oxidative stress markers and alternative definitions of sleep quality to better understand the interactions among these pathways. 


\section{Abbreviations}

BMl: Body mass index; Cl: Confidence interval; CRP: C-reactive protein; IL6: Interleukin-6; NHANES: National health and nutrition examination survey; RNS: Reactive nitrogen species; ROS: Reactive oxygen species; TNF: Tumour necrosis factor

\section{Availability of data and materials}

The datasets generated and/or analysed during the current study are publicly available in the NHANES repository, https://wwwn.cdc.gov/nchs/nhanes/

\section{Authors' contributions}

VT and TK participated in the conception, design, and acquisition of data. TK performed the analyses. All authors worked together to interpret the results and AR drafted the manuscript. All authors read and approved the final manuscript.

\section{Ethics approval and consent to participate}

Ethical approval was provided by the National Center for Health Studies Ethics Review Board for the NHANES, and informed consent was obtained from all participants.

\section{Consent for publication}

Not applicable.

\section{Competing interests}

Valerie Taylor is an associate editor at BMC Obesity.

\section{Publisher's Note}

Springer Nature remains neutral with regard to jurisdictional claims in published maps and institutional affiliations.

\section{Author details}

${ }^{1}$ Faculty of Medicine, University of Toronto, Toronto, Canada. ${ }^{2}$ Department of Epidemiology, Biostatistics, and Occupational Health, McGill University, Montreal, Canada. 'Women's College Research Institute, University of Toronto, Toronto, Canada. ${ }^{4}$ Women's College Hospital, 76 Grenville Street Room E947, Toronto, ON M5S 1B2, Canada.

Received: 13 March 2018 Accepted: 21 August 2018

Published online: 03 December 2018

\section{References}

1. Faith MS, Butryn M, Wadden TA, Fabricatore A, Nguyen AM, Heymsfield SB. Evidence for prospective associations among depression and obesity in population-based studies. Obes Rev. 2011;12:438-53.

2. Luppino FS, de Wit LM, Bouvy PF, Stijnen T, Cuijpers P, Penninx BWJH, et al. Overweight, obesity, and depression. Arch Gen Psychiatry. 2010;67:220-9.

3. Forhan M, Gill SV. Obesity, functional mobility and quality of life. Best Pract Res Clin Endocrinol Metab. 2013;27:129-37.

4. Chen EY, Bocchieri-Ricciardi LE, Munoz D, Fischer S, Katterman S, Roehrig M, et al. Depressed mood in class III obesity predicted by weight-related stigma. Obes Surg. 2007:17:669-71. https://doi.org/10.1007/s11695-007-9112-4.

5. Neogi T. The epidemiology and impact of pain in osteoarthritis. Osteoarthr Cartil. 2013;21:1145-53

6. Marcus DA. Obesity and the impact of chronic pain. Clin J Pain. 2004;20:186-91.

7. Sharma L, Lou C, Cahue S, Dunlop DD. The mechanism of the effect of obesity in knee arthritis: the mediating role of malalignment. Arthritis Rheum. 2000;43:568-75.

8. Staiano AE, Marker AM, Martin CK, Katzmarzyk PT. Physical activity, mental health, and weight gain in a longitudinal observational cohort of nonobese young adults. Obesity. 2016:24:1969-75.

9. Shelton RC, Falola M, Li L, Zajecka J, Fava M, Papakostas Gl. The proinflammatory profile of depressed patients is (partly) related to obesity. J Psychiatr Res. 2015;70:91-7.

10. Shelton RC, Miller AH. Inflammation in depression: is adiposity a cause? Dialogues Clin Neurosci. 2011;13:41-54.

11. Raison CL, Capuron L, Miller AH. Cytokines sing the blues: inflammation and the pathogenesis of depression. Trends Immunol. 2006:27:24-31.

12. Raison CL, Rutherford RE, Woolwine BJ, Shuo C, Schettler P, Drake DF, et al. A randomized controlled trial of the tumor necrosis factor antagonist infliximab for treatment-resistant depression. JAMA Psychiatry. 2013;70:31-41.
13. Persoons P, Vermeire S, Demyttenaere K, Fischler B, Vandenberghe J, Van Oudenhove $L$, et al. The impact of major depressive disorder on the shortand long-term outcome of Crohn's disease treatment with infliximab. Aliment Pharmacol Ther. 2005:22:101-10.

14. Odegaard Jl, Chawla A. Alternative macrophage activation and metabolism. Annu Rev Pathol. 2011;6:275-97.

15. Sies $\mathrm{H}$. Role of reactive oxygen species in biological processes. Klin Wochenschr. 1991;69:965-8.

16. Keaney JF, Larson MG, Vasan RS, Wilson PWF, Lipinska I, Corey D, et al. Obesity and systemic oxidative stress: clinical correlates of oxidative stress in the Framingham study. Arterioscler Thromb Vasc Biol. 2003;23:434-9.

17. Liu T, Zhong S, Liao X, Chen J, He T, Lai S, et al. A meta-analysis of oxidative stress markers in depression. PLoS One. 2015:10:1-17.

18. Gawryluk JW, Wang J-F, Andreazza AC, Shao L, Yatham LN, Young LT. Prefrontal cortex glutathione S-transferase levels in patients with bipolar disorder, major depression and schizophrenia. Int J Neuropsychopharmacol. 2011:14:1069-74.

19. Gałecki P, Szemraj J, Bieńkiewicz M, Florkowski A, Gałecka E. Lipid peroxidation and antioxidant protection in patients during acute depressive episodes and in remission after fluoxetine treatment. Pharmacol Rep. 2009;61:436-47.

20. Motivala SJ, Irwin MR. Sleep and immunity: cytokine pathways linking sleep and health outcomes. Curr Dir Psychol Sci. 2007;16:21-5.

21. Meier-Ewert HK, Ridker PM, Rifai N, Regan MM, Price NJ, Dinges DF, et al. Effect of sleep loss on C-reactive protein, an inflammatory marker of cardiovascular risk. J Am Coll Cardiol. 2004;43:678-83.

22. Vgontzas AN, Zoumakis E, Bixler EO, Lin HM, Follett H, Kales A, et al. Adverse effects of modest sleep restriction on sleepiness, performance, and inflammatory cytokines. J Clin Endocrinol Metab. 2004:89:2119-26.

23. Howren MB, Lamkin DM, Suls J. Associations of depression with C-reactive protein, IL-1, and IL-6: a meta-analysis. Psychosom Med. 2009:71:171-86.

24. Opp MR. Cytokines and sleep. Sleep Med Rev. 2005:9:355-64.

25. Mullington J, Korth C, Hermann DM, Orth A, Galanos C, Holsboer F, et al. Dose-dependent effects of endotoxin on human sleep. Am J Physiol Regul Integr Comp Physiol. 2000;278:R947-55.

26. Heinzelmann M, Lee H, Rak H, Livingston W, Barr T, Baxter T, et al. Sleep restoration is associated with reduced plasma C-reactive protein and depression symptoms in military personnel with sleep disturbance after deployment. Sleep Med. 2014;15:1565-70.

27. Nedeltcheva AV, Kilkus JM, Imperial J, Schoeller DA, Penev PD. Insufficient sleep undermines dietary efforts to reduce adiposity. Ann Intern Med. 2010;153:435-41.

28. National Center for Health Statistics. NHANES - About the National Health and Nutrition Examination Survey 2011. http://www.cdc.gov/nchs/nhanes/ about_nhanes.htm (accessed 6 Aug 2016).

29. Kanagasabai T, Ardern Cl. Inflammation, oxidative stress, and antioxidants contribute to selected sleep quality and Cardiometabolic health relationships: a cross-sectional study. Mediat Inflamm. 2015:2015:1-11.

30. Kroenke K, Spitzer RL, Williams JBW. The PHQ-9: validity of a brief depression severity measure. J Gen Intern Med. 2001:16:606-13.

31. Liu Y, Al-Sayegh H, Jabrah R, Wang W, Yan F, Zhang J. Association between C-reactive protein and depression: modulated by gender and mediated by body weight. Psychiatry Res. 2014;219:103-8.

32. Spitzer RL, Kroenke K, Williams JW. The patient health questionnaire primary care study group. Validation and utility of a self-report version of prime-md: the phq primary care study. JAMA. 1999:282:1737-44.

33. Ferguson KK, Loch-Caruso R, Meeker JD. Exploration of oxidative stress and inflammatory markers in relation to urinary phthalate metabolites: NHANES 1999-2006. Environ Sci Technol. 2012;46:477-85.

34. Kobrosly R, van Wijngaarden E. Associations between immunologic, inflammatory, and oxidative stress markers with severity of depressive symptoms: an analysis of the 2005-2006 National Health and nutrition examination survey. Neurotoxicology. 2010;31:126-33.

35. Zipf G, Chiappa M, Porter K, Ostchega Y, Lewis B, Dostal J. National Health and Nutrition examination survey: plan and operations, 1999 - 2010. Natl Cent Heal Stat Vital Heal Stat. 2013;56:1

36. National Center for Health Statistics. NHANES 2005-2006 Lab Methods CDC 2007. https://wwwn.cdc.gov/nchs/nhanes/continuousnhanes/labmethods. aspx?BeginYear=2005 (accessed 6 Aug 2016).

37. Kanagasabai T, Nie JX, Mason C, Ardern Cl. Metabolic syndrome and prevalent any-site, prostate, breast and Colon cancers in the US adult population: NHANES 1999-2010. J Metab Synd. 2014;3:2167-943. 
38. Ford ES. Lung function, 25-hydroxyvitamin D concentrations and mortality in US adults. Eur J Clin Nutr. 2015;69:572-8.

39. Vincent HK, Taylor AG. Biomarkers and potential mechanisms of obesityinduced oxidant stress in humans. Int J Obes. 2006;30:400-18.

40. Miller GE, Stetler CA, Carney RM, Freedland KE, Banks WA. Clinical depression and inflammatory risk markers for coronary heart disease. Am J Cardiol. 2002;90:1279-83.

41. Dowlati Y, Herrmann N, Swardfager W, Liu H, Sham L, Reim EK, et al. A metaanalysis of cytokines in major depression. Biol Psychiatry. 2010;67:446-57.

42. Miller GE, Freedland KE, Carney RM, Stetler CA, Banks WA. Pathways linking depression, adiposity, and inflammatory markers in healthy young adults. Brain Behav Immun. 2003;17:276-85.

43. Klein AS, Bulkley GB, Bao C, Noble PW, Lane MD, Diehl AM, et al. Leptin regulates proinflammatory immune responses tributes to several of the major complications of obe. FASEB J. 1998;12:57-65.

44. Irwin MR, Miller AH. Depressive disorders and immunity: 20 years of progress and discovery. Brain Behav Immun. 2007;21:374-83.

45. Raison $\mathrm{CL}$, Miller $\mathrm{AH}$. When not enough is too much: the role of insufficient glucocorticoid signaling in the pathophysiology of stress-related disorders. Am J Psychiatry. 2003;160:1554-65.

46. Pfäfflin A, Schleicher E. Inflammation markers in point-of-care testing (POCT). Anal Bioanal Chem. 2009;393:1473-80.

47. Ladwig KH, Marten-Mittag B, Löwel H, Döring A, Koenig W. Influence of depressive mood on the association of CRP and obesity in 3205 middle aged healthy men. Brain Behav Immun. 2003;17:268-75. https://doi.org/10. 1016/S0889-1591(03)00056-4.

48. Black CN, Bot M, Scheffer PG, Cuijpers P, Penninx BWJH. Is depression associated with increased oxidative stress? A systematic review and metaanalysis. Psychoneuroendocrinology. 2015;51:164-75.

49. Berk M, Williams $L$, Jacka FN, O’Neil A, J a P, Moylan S, et al. So depression is an inflammatory disease, but where does the inflammation come from? BMC Med. 2013;11:200.

50. Maes M, Kubera M, Obuchowiczwa E, Goehler L, Brzeszcz J. Depression's multiple comorbidities explained by (neuro)inflammatory and oxidative \& nitrosative stress pathways. Neuroendocrinol Lett. 2011;32:7-24.

51. Marazziti D, Rutigliano G, Baroni S, Landi P, Dell'osso L. Metabolic syndrome and major depression. CNS Spectr. 2013;19:1-12.

52. Furukawa S, Fujita T, Shumabukuro M, Iwaki M, Yamada Y, Makajima Y, et al. Increased oxidative stress in obesity and its impact on metabolic syndrome. J Clin Invest. 2004;114:1752-61.

53. Vincent HK, Innes KE, Vincent KR. Oxidative stress and potential interventions to reduce oxidative stress in overweight and obesity. Diabetes Obes Metab. 2007;9:813-39.

54. Power ML, Schulkin J. Sex differences in fat storage, fat metabolism, and the health risks from obesity: possible evolutionary origins. Br J Nutr. 2008;99: 931-40.

55. Kamper EF, Chatzigeorgiou A, Tsimpoukidi O, Kamper M, Dalla C, Pitychoutis $P$, et al. Sex differences in oxidant/antioxidant balance under a chronic mild stress regime. Physiol Behav. 2009;98:215-22.

56. Ramsey JM, Cooper JD, Bot M, Guest PC, Lamers F, Weickert CS, et al. Sex differences in serum markers of major depressive disorder in the Netherlands study of depression and anxiety (NESDA). PLoS One. 2016;11:e0156624.

57. Abdelkrim K, Ali T, Sina K, Kazem B, Mohammed D, Naser E. Gender-specific Association of Oxidative Stress and Inflammation with cardiovascular risk factors in Arab population. Mediat Inflamm. 2015;2015:11.

58. Matsushita M, Kumano-Go T, Suganuma N, Adachi H, Yamanura S, Morishima $\mathrm{H}$, et al. Do depressive symptoms correlate with oxidative stress in a sample of healthy college students? Eur J Psychiat. 2010;24:63-9.

59. Kodydkova J, Vavrova L, Zeman M, Jirak R, Macasek J, SStankova B, et al. Antioxidative enzymes and increased oxidative stress in depressive women. Clin Biochem. 2009:42:1368-74.

60. Wiener C, Rassier GT, Kaster MP, Jansen K, Pinheiro RT, Klamt F, et al. Gender-based differences in oxidative stress parameters do not underlie the differences in mood disorders susceptibility between sexes. Eur Psychiatry. 2014:29:58-63.

61. Gariballa S, Kosanovic M, Yasin J, El EA. Oxidative damage and inflammation in obese diabetic emirati subjects. Nutrients. 2014;6:4872-80.

62. Irwin MR, Olmstead R, Carroll JE. Archival report sleep disturbance, sleep duration, and Inflammation : a systematic review and meta-analysis of cohort studies and experimental sleep deprivation. Biol Psychiatry. 2015;80:1-13.
63. Liu R, Liu X, Zee PC, Hou L, Zheng Z, Wei Y, et al. Association between sleep quality and C-reactive protein: results from national health and nutrition examination survey, 2005-2008. PLoS One. 2014;9:2005-8.

64. Prather A, Vogelzangs N, Penninx BWJH. Sleep duration, insomnia, and markers of systemic inflammation: results from the Netherlands study of depression and anxiety (NESDA). J Psychiatr Res. 2015;60:95-102.

65. Sutcigil L, Oktenli C, Musabak U, Bozkurt A, Cansever A, Uzun O, et al. Pro- and anti-inflammatory cytokine balance in major depression: effect of sertraline therapy. Clin Dev Immunol. 2007;2007:6-11.

66. Bilici M, Efe H, Koroglu MA, Uydu HA, Bekaroglu M, Deger O. Antioxidative enzyme activities and lipid peroxidation in major depression: alterations by antidepressant treatments. J Affect Disord. 2001;64:43-51.
Ready to submit your research? Choose BMC and benefit from:

- fast, convenient online submission

- thorough peer review by experienced researchers in your field

- rapid publication on acceptance

- support for research data, including large and complex data types

- gold Open Access which fosters wider collaboration and increased citations

- maximum visibility for your research: over $100 \mathrm{M}$ website views per year

At $\mathrm{BMC}$, research is always in progress.

Learn more biomedcentral.com/submissions 